\title{
Good Smile, Healthy Brain?
}

Souvik Sen* and Lauren Dennis Giamberardino

Department of Neurology, University of South Carolina School of Medicine, Columbia, South Carolina, USA

*Corresponding author: Souvik Sen, Department of Neurology, University of South Carolina School of Medicine, Columbia, South Carolina, USA, Tel: (803) 545-6073; E-mail: Souvik.Sen@uscmed.sc.edu

Rec date: April 25, 2016; Acc date: April 27, 2016; Pub date: April 29, 2016

Copyright: $\odot 2016$ Sen S, et al. This is an open-access article distributed under the terms of the Creative Commons Attribution License, which permits unrestricted use, distribution, and reproduction in any medium, provided the original author and source are credited.

\section{Short Communication}

Periodontal disease is an infectious disease causes my microorganisms resulting in the corrosion of tissues around the tooth surface and soft tissue lining the mouth, loss of connective tissue attachment, breakdown of bone that holds the teeth, and tooth loss [1]. The symptoms are bleeding gum (gingivitis), gingival pockets and bone loss (periodontal disease) leading to tooth mobility and eventually tooth loss. In the United States, periodontal disease is very prevalent and becomes more prevalent in aging populations. According to a recent survey in the United States, approximately half of adults have some level of periodontal disease and almost $10 \%$ of those have severe disease [2]. Periodontal disease is related to bacterial infection related systemic inflammation, which is implicated in the etiology of atherosclerosis leading to stroke, heart attack and other cardiovascular disease [3]. Epidemiological studies have suggested a possible association between periodontal disease and stroke. The linkage is derived from cross-sectional, case-control and cohort studies [4-16]. Analyses from recent, large-cohort study suggest a stepwise relationship between tooth loss, stroke, cardiovascular death, and allcause mortality in individuals with stable coronary artery disease [17]. If these relationships are in fact a direct, causal relationship, these associations would have great implication due to the potential of reducing the risk of major adverse cardiovascular events, including stroke, by preventing or treating periodontal disease.

Individual studies have many limitations, including the use of many differing definitions of periodontal disease, consideration of potential cofounders such as socioeconomic status, and low statistical power for detecting cardiovascular events such as stroke or transient ischemic attacks (TIA). Many of these issues were addressed in a prospective cohort study which evaluated the association between the presence of periodontal disease and recurrent vascular events in stroke/TIA patients [18]. One-hundred-six ischemic stroke or TIA patients were recruited at a single centre. At enrollment, patients with high periodontal disease (defined as the highest tertile of attachment loss $\geq$ $5 \mathrm{~mm}$ and coinciding with initial and severe periodontal disease) tended to be older, male, African-American, and to have lower education level and annual income; but, did not differ significantly by age, or prevalence of traditional risk factors including smoking, hypertension and cholesterol levels. Of these patients, 27 (26\%) had recurrent composite vascular events over a median follow-up period of 24 months and $40(38 \%)$ showed high periodontal disease. The associated Kaplan-Meier curve exhibited a decidedly higher proportion of patients with high periodontal disease experienced composite events of stroke/TIA/MI/death ( $47 \%$ compared to those who did not exhibit periodontal disease 19\%; $\mathrm{p}=0.02$ ). Periodontal disease was independently associated with composite vascular events (hazard ratio 2.8, 95\% CI, 1.2-6.5), after adjustment for potential confounders, the association with high periodontal disease remained significant (hazard ratio 2.8, 95\% CI, 1.0-8.0). This study determined that further studies are needed to assess whether treatment of periodontal disease can reduce the rate of recurrent vascular events in patients with ischemic stroke or TIA [18].

Several mechanisms have been suggested in an attempt to describe the association between periodontal disease and stroke. Periodontal disease has been suggested to be the result of a complex interaction between infection and the immune response, resulting in an inflammatory response. Inflammatory markers have been associated with vascular events in stroke and TIA patients. Therefore the inflammatory response to periodontal disease may be the link to the association of inflammatory response and vascular events. During normal daily activities such as chewing food, microorganisms are able to enter the bloodstream creating a local and systemic inflammation resulting in an immune response $[19,20]$. Periodontal disease is most likely mediated by a host-bacterial interaction and the host's response to the presence of microorganisms likely determines the periodontal disease [21]. Treatment of periodontal disease also temporarily causes a release of inflammatory markers due to the release of bacteria or inflammatory cytokines into the blood stream [22], however, in time the levels of inflammatory markers declines and endothelial function is improved [22,23]. The Oral Infections and Vascular Disease Epidemiology Study (INVEST) study suggests an association between carotid intimal-medial thickness and the volume of pathogenic bacteria during dental examination [24]. Additional studies have reported reduced carotid intimal-medial thickness six months post intensive periodontal disease treatment [25].

Evidence suggests that inflammation and immune response are linked to the development of atherosclerosis, a major risk factor in cardiovascular and cerebrovascular disease. Thus, the systemic inflammatory or immune response to periodontal disease may also increase cardiovascular risk. Pathogenic bacteria can enter the mouth via the blood stream, attach to atherosclerotic plaque and can develop into inflammation, stimulating an immune response within said atherosclerotic plaque. Studies have discovered a wide range of pathogenic bacteria in the oral biome and DNA from these bacteria has also been detected in atherosclerotic plaque [26,27]. In animal models, studies have found an accumulation of cholesterol esters and inflammatory mediators in association with an increase in atherosclerotic plaque due to infection with Porphyromonas gingivalis [28]. In humans, accumulation of serum IgA antibodies to $P$ gingivalis may be linked to an increase in stroke risk as these antibodies are higher in stroke patients than in the controls [29].

Though there appears to be unequivocal pathophysiological evidence to support the hypothesized mechanisms, it is also plausible that the association between periodontal disease and stroke is in fact not causal. Part of the association can be explained by adjustment for cardiovascular risk factors or cofounders to include smoking, diabetes 
mellitus, increasing age, and poor socioeconomic conditions; all risk factors for both periodontal disease and cardiovascular disease, specifically stroke [1]. The measurement of these confounders can often be inaccurate, and some confounders may even go unidentified. For example, poor diet, non-compliance with medications, poor health habits, and low socioeconomic status could each increase the risk of both periodontal disease and stroke; however these are rarely measured in most studies. Deciphering the evidence of multiple common risk factors between periodontal disease and cardiovascular disease is highly relevant to understanding the possible link between atherosclerosis and periodontal disease. Furthermore, this information could establish further preventive measures like smoking cessation, reducing obesity, and improving glucose tolerance, which could positively benefit both periodontal disease and cardiovascular disease.

One may ask, should the treatment of periodontal disease be a recommendation or guideline in the effort to reduce the risk of stroke? Evidence from randomized controlled trials have established that intensive periodontal treatment reduces the proportion of individuals with periodontal organisms, systemic inflammation, high blood pressure, improves lipid profile, and endothelial dysfunction [30-32]. Therefore, the treatment of periodontal disease could plausibly reduce stroke incidence, but this has not, to the best of our knowledge, been tested in a randomized clinical trial. A recent retrospective cohort study retrieved from the Taiwanese National Health Insurance Research Database administrative data suggest that maintaining periodontal health by receiving dental prophylaxis and PD treatment can help reduce the incidence of ischemic stroke [33]. A US-based epidemiological study showed that improvement in periodontal disease status lead to improvement in carotid atherosclerosis [34]. A recent prospective longitudinal study conducted in Germany showed that usage of floss and interdental brushing independently reduced the risk of stroke, transient ischemic attack (TIA), myocardial infarction (MI) and cardiovascular death in patients with coronary heart disease [35]. In a recent prospective cohort study conducted among ischemic stroke survivors, we found that moderate to severe periodontal disease was correlated with an approximately three-fold higher risk of recurrent vascular events at two years [18]. The effect of treatment of periodontal disease on recurrent vascular events in stroke/transient ischemic attack (TIA) patients has not been studied. To ascertain the possible effect of periodontal treatment on major cardiovascular disease such as stroke, a large clinical trial statistically powered to detect an effect. Because periodontal disease is a chronic disease, a long-term approach would be more adapt than a single treatment study in achieving continued improvement in periodontal disease and systemic health. Periodontal disease can be treated my increased frequency of tooth brushing and flossing, root scaling and planning and regular dental visits to a dentist or dental hygienist. These are current guidelines for good oral health but are associated with possible benefits for stroke patients. Stroke patients with periodontal disease who receive regular dental treatment are less likely to suffer from cerebrovascular disease such as stroke. However, evaluation the effect of treatment and intervention for periodontal disease on reducing the cardiovascular disease in stroke patients presents its own challenges.

Scientific statements related to stroke have not addressed the role of periodontal disease treatment in stroke prevention [35,36]. In 2012, an American Heart Association scientific statement concluded no evidence that periodontal disease causes atherosclerosis and cautioned that any statements suggesting an association between the two or those certain periodontal treatments may be beneficial, are groundless [3]. Today, this argument against the association between periodontal disease and cerebrovascular disease remains appropriate to the stroke population. Although several studies allude to the association between periodontal disease and stroke, the fact that the association can largely be explained by common risk factors or cofounders is important because it provides further evidence and justification that strategies to reduce cardiovascular and stroke risk, such as the American Heart Association's Life's Simple 7, are also likely to benefit other determinants of good health.

\section{References}

1. Pihlstrom BL, Michalowicz BS, Johnson NW (2005) Periodontal diseases. Lancet 366: 1809-1820.

2. Eke PI, Dye BA, Wei L, Thornton-Evans GO, Genco RJ (2012) CDC Periodontal disease Surveillance workgroup: James Beck (University of North Carolina, Chapel Hill, USA), Gordon Douglass (Past President, American Academy of Periodontology), Roy Page (University of Washin. Prevalence of periodontitis in adults in the United States: 2009 and 2010. J Dent Res 91:914-920.

3. Lockhart PB, Bolger AF, Papapanou PN, Osinbowale O, Trevisan M, et al. (2012) American Heart Association Rheumatic Fever, Endocarditis, and Kawasaki Disease Committee of the Council on Cardiovascular Disease in the Young, Council on Epidemiology and Prevention, Council on Peripheral Vascular Disease, and Council on Clinical Cardiology. Periodontitis and atherosclerotic vascular disease: does the evidence support an independent association?: a scientific statement from the American Heart Association. Circulation 125: 2520-2544.

4. Lee HJ, Garcia RI, Janket SJ, Jones JA, Mascarenhas AK, et al. (2006) The association between cumulative periodontal disease and stroke history in older adults. J Periodontol 77: 1744-1754.

5. Grau AJ, Becher H, Ziegler CM, Lichy C, Buggle F, et al. (2004) Periodontal disease as a risk factor for ischemic stroke. Stroke 35: 496-501.

6. Sim SJ, Kim HD, Moon JY, Zavras AI, Zdanowicz J, et al. (2008) Periodontitis and the risk for non-fatal stroke in Korean adults. J Periodontol 79: 1652-1658.

7. Kim HD, Sim SJ, Moon JY, Hong YC, Han DH (2010) Association between periodontitis and hemorrhagic stroke among Koreans: a case-control study. J Periodontol 81: 658-665.

8. Pradeep AR, Hadge P, Arjun Raju P, Shetty SR, Shareef K, et al. (2010) Periodontitis as a risk factor for cerebrovascular accident: a case-control study in the Indian population. J Periodontal Res 45: 223-228.

9. Wu T, Trevisan M, Genco RJ, Dorn JP, Falkner KL, et al. (2000) Periodontal disease and risk of cerebrovascular disease: the first national health and nutrition examination survey and its follow-up study. Arch Intern Med 160: 2749-2755.

10. Howell TH, Ridker PM, Ajani UA, Hennekens CH, Christen WG (2001) Periodontal disease and risk of subsequent cardiovascular disease in U.S. male physicians. J Am Coll Cardiol 37: 445-450.

11. Abnet CC, Qiao Y-L, Dawsey SM, Dong Z-W, Taylor PR, et al. (2005) Tooth loss is associated with increased risk of total death and death from upper gastrointestinal cancer, heart disease, and stroke in a Chinese populationbased cohort. Int J Epidemiol 34: 467-474.

12. Tu YK, Galobardes B, Smith GD, McCarron P, Jeffreys M, et al. (2007) Associations between tooth loss and mortality patterns in the Glasgow Alumni Cohort. Heart 93: 1098-1103.

13. Heitmann BL, Gamborg M (2008) Remaining teeth, cardiovascular morbidity and death among adult Danes. Prev Med 47: 156-160.

14. Jimenez M, Krall EA, Garcia RI, Vokonas PS, Dietrich T (2009) Periodontitis and incidence of cerebrovascular disease in men. Ann Neurol 66: 505-512.

15. Holmlund A, Holm G, Lind L (2010) Number of teeth as a predictor of cardiovascular mortality in a cohort of 7,674 subjects followed for 12 years. J Periodontol 81: 870-876. 
16. Morrison HI, Ellison LF, Taylor GW (1999) Periodontal disease and risk of fatal coronary heart and cerebrovascular diseases. J Cardiovasc Risk 6: 7-11.

17. Vedin O, Hagstrom E, Budaj A, Denchev S, Harrington RA, et al. (2015) Tooth loss is independently associated with poor outcomes in stable coronary heart disease. Eur J Prev Cardiol.

18. Sen S, Sumner R, Hardin J, Barros S, Moss K, et al. "Periodontitis and recurrent vascular events in stroke/transient ischemic attack patients." Journal of Stroke and Cerebrovascular Diseases 22.8 (2013): 1420-427.

19. Kinane DF, Riggio MP, Walker KF, MacKenzie D, Shearer B (2005) Bacteraemia following periodontal procedures. J Clin Periodontol 32: 708-713.

20. Lockhart PB, Brennan MT, Thornhill M, Michalowicz BS, Noll J, et al. (2009) Poor oral hygiene as a risk factor for infective endocarditis-related bacteremia. J Am Dent Assoc 140: 1238-1244.

21. Hein C, Cobb C, lacopino A (2007) Report of the Independent Panel of Experts of the Scottsdale Project. Grand Rounds Oral Systemic Med 1-27.

22. Tonetti MS, D'Aiuto F, Nibali L, Donald A, Storry C, et al. (2007) Treatment of periodontitis and endothelial function. N Engl J Med 356: 911-920.

23. Teeuw WJ, Slot DE, Susanto H, Gerdes VE, Abbas F, et al. (2014) Treatment of periodontitis improves the atherosclerotic profile: a systematic review and meta-analysis. J Clin Periodontol 41: 70-79.

24. Desvarieux M, Demmer RT, Rundek T, Boden-Albala B, Papapanou PN, et al. (2003) Oral Infections and Vascular Disease Epidemiology Study (INVEST). Relationship between periodontitis, tooth loss, and carotid artery plaque: the Oral Infections and Vascular Disease Epidemiology Study (INVEST). Stroke 34:2120-2125.

25. Piconi S, Trabattoni D, Luraghi C, Perilli E, Borelli M, et al. (2009) Treatment of periodontal disease results in improvements in endothelial dysfunction and reduction of the carotid intima-media thickness. FASEB J 23:1196-1204.

26. Ott SJ, El Mokhtari NE, Musfeldt M, Hellmig S, Freitag S, et al. (2006) Detection of diverse bacterial signatures in atherosclerotic lesions of patients with coronary heart disease. Circulation 113: 929-937.

27. Fernandes CP, Oliveira FA, Silva PG, Alves AP, Mota MR, et al. (2014) Molecular analysis of oral bacteria in dental biofilm and atherosclerotic plaques of patients with vascular disease. Int J Cardiol 174: 710-712.
28. Hayashi C, Viereck J, Hua N, Phinikaridou A, Madrigal AG, et al. (2011) Porphyromonas gingivalis accelerates inflammatory atherosclerosis in the innominate artery of ApoE deficient mice. Atherosclerosis 215: 52-59.

29. Pussinen PJ, Alfthan G, Rissanen H, Reunanen A, Asikainen S, et al. (2004) Antibodies to periodontal pathogens and stroke risk. Stroke 35: 2020-2023.

30. Ready D, D'Aiuto F, Spratt DA, Suvan J, Tonetti MS, et al. (2008) Disease severity associated with presence in subgingival plaque of Porphyromonas gingivalis, Aggregatibacter actinomycetemcomitans, and Tannerella forsythia, singly or in combination, as detected by nested multiplex PCR. J Clin Microbiol 46: 3380-3383.

31. D'Aiuto F, Parkar M, Nibali L, Suvan J, Lessem J, et al. (2006) Periodontal infections cause changes in traditional and novel cardiovascular risk factors: results from a randomized controlled clinical trial. Am Heart J 151: 977-984.

32. Lee YL, Hu HY, Huang N, Hwang DK, Chou P, et al. (2013) Dental prophylaxis and periodontal treatment are protective factors to ischemic stroke. Stroke 44: 1026-1030.

33. Desvarieux M, Demmer RT, Jacobs DR, Papapanou PN, Sacco RL, et al. (2013) "Changes in clinical and microbiological periodontal profiles relate to progression of carotid intima-media thickness: The Oral Infections and Vascular Disease Epidemiology Study. Journal of the American Heart Association 2:6.

34. Reichert S, Schlitt A, Beschow V, Lutze A, Lischewski S, et al. (2015) Use of floss/interdental brushes is associated with lower risk for new cardiovascular events among patients with coronary heart disease. J Periodontal Res 50: 180-188.

35. Meschia JF, Bushnell C, Boden-Albala B, Braun LT, Bravata DM, et al. (2014) Guidelines for the primary prevention of stroke. Stroke 45: 3754-832.

36. KernanWN, Ovbiagele B, Black HR, Bravata DM, Chimowitz MI, et al (2014) Guidelines for the prevention of stroke in patients with stroke and transient ischemic attack: A guideline for healthcare professionals from the American Heart Association/American Stroke Association. Stroke 45: 2160-236. 